\title{
Determinants of phosphatidylinositol-4-phosphate 5-kinase type I Y 90 uropod location in T-lymphocytes and its role in uropod formation
}

We have previously identified phosphatidylinositol-4-phosphate 5-kinase type I (PIPKI) g 90 as a T cell uropod component. However, the molecular determinants and functional consequences of its localization remain unknown. In this report, we seek to better understand the mechanisms involved in PIPKI $\gamma 90$ uropod targeting and the role that PIPKI $\gamma 90$ plays in $\mathrm{T}$ cell uropod formation. During $\mathrm{T}$ cell activation, PIPKI $\gamma 90$ cocaps with the membrane microdomain-associated proteins flotillin-1 and -2 and accumulates in the uropod. We report that the C-terminal 26 amino acid extension of PIPKI $y$ 90 is required for its localization to the uropod. We further use T cells from PIPKI $\gamma 90^{-/-}$mice and human T cells expressing a kinase-dead PIPKI $\gamma 90$ mutant to examine the role of PIPKI g 90 in T cell uropod formation. We find that PIPKI $\gamma 90$ deficient $\mathrm{T}$ cells have elongated uropods on ICAM-1. Moreover, in human T cells overexpression of PIPKI $\gamma$ 87, a naturally occurring isoform lacking the last 26 amino acids, suppresses uropod formation and impairs capping of uropod proteins such as flotillins. Transfection of human T cells with a dominant-negative mutant of flotillin-2 in turn attenuates capping of PIPKI $\gamma$ 90. Our data contribute to the understanding of the molecular mechanisms that regulate $\mathrm{T}$ cell uropod formation. 


\section{1}

1 Determinants of phosphatidylinositol-4-phosphate 5-kinase type I $\gamma 90$ uropod location in

2 T-lymphocytes and its role in uropod formation

3

4 Lucia Mathis $^{1}$, Sarah Wernimont ${ }^{2}$, Sarah Affentranger ${ }^{1}$, Anna Huttenlocher $^{2}$, Verena Niggli ${ }^{1}$

5

6

7

$8{ }^{1}$ Institute of Pathology, University of Bern, CH-3010 Bern, Switzerland; ${ }^{2}$ Departments of Medical

9 Microbiology and Immunology and Pediatrics, University of Wisconsin, Madison, WI 53706,

10 United States of America

11

12

13 Address for correspondence: Verena Niggli, Institute of Pathology, University of Bern,

14 Murtenstrasse 31, CH-3010 Bern, Switzerland; phone +41 3163287 44; E-mail:

15 verena.niggli@pathology.unibe.ch

16

17 Abbreviations: $\mathrm{PI}(4,5) \mathrm{P}_{2}$, phosphatidylinositol 4,5 bisphosphate; PIPKI,

18 phosphatidylinositol-4-phosphate 5-kinase type I; ICAM-1, intercellular adhesion molecule 1;

19 KD, kinase-dead; P-ERM, phosphorylated ezrin/radixin/moesin proteins; PFA,

20 paraformaldehyde; rhICAM-1, recombinant human ICAM-1; rmICAM-1, recombinant murine

21 ICAM-1; SDF-1, stromal cell-derived factor-1

22 
2

\section{Introduction}

24 Migrating leukocytes establish a polarized morphology with an actin-rich leading edge and a 25 uropod protruding from the trailing edge. This polarization is essential for efficient crawling. The 26 uropod is a plasma membrane protrusion that contains specific organelles along with cytoskeletal, 27 adhesion and signaling proteins (Serrador et al., 1997; Sánchez-Madrid \& Serrador, 2009).

28 Flotillins, membrane microdomain scaffolding proteins, are also enriched in leukocyte uropods 29 and are involved in uropod formation (Rossy et al., 2009; Ludwig et al., 2010; Affentranger et al., 30 2011; Baumann, Affentranger \& Niggli, 2012). Recent in vivo data using inhibition of uropod 31 formation by suppressing Rho-kinase activity suggest that the uropod is especially important for 32 T cell migration through constricted spaces (Soriano et al., 2011).

33 During leukocyte migration, Rac2 mediated actin polymerization at the leading edge drives cell 34 protrusion, while RhoA activation controls myosin II dependent uropod retraction (Xu et al., 35 2003). This process of protrusion and retraction is exquisitely coordinated to support efficient cell 36 migration. Phosphoinositides are important signaling lipids regulating these processes. Research 37 has focused especially on phosphatidylinositol $(4,5)$ bisphosphate $\left(\mathrm{PI}(4,5) \mathrm{P}_{2}\right)$ and 38 phosphatidylinositol $(3,4,5)$ trisphosphate. Production of the former lipid is regulated by 39 phosphatidylinositol-4-phosphate 5-kinase type I (PIPKI) isoforms and that of the latter lipid by phosphoinositide 3-kinase (Ward, Westwick \& Harris, 2011; Sun et al., 2013). Previously, we identified the enzyme PIPKI 90 enriched in the neutrophil and the T cell uropod (Lokuta et al., 2007). PIPKI $\gamma 90$ has been implicated in the regulation of integrin activity and endocytosis (Calderwood et al., 2004; Bairstow et al., 2006; Sekine et al., 2007). Two isoforms of PIPKI $\gamma$ that differ by a 26 amino acid C-terminal extension are expressed in T cells; a 635 amino acid (87 $\mathrm{kDa}$ ) isoform, PIPKI $\gamma 87$, and a 661 amino acid (90 kDa) isoform, PIPKI $\gamma 90$ (Wernimont et al., 2010). Neutrophils lacking both PIPKI $\gamma$ isoforms have impaired neutrophil adhesion and trafficking to sites of infection. PIPKI 87 lacks the sequence required for uropod targeting as observed in neutrophils (Xu et al., 2010). In T cells, siRNA knockdown of both PIPKI $\gamma$ isoforms is associated with decreased integrin mediated $\mathrm{T}$ cell adhesion in response to chemokine 
3

50 signaling (Bolomini-Vittori et al., 2009), whereas T cells from PIPKI $\gamma 90$ deficient mice have

51 increased integrin mediated adhesion in response to $\mathrm{T}$ cell receptor-mediated signaling

52 (Wernimont et al., 2010), indicating that the two isoforms have distinct functions.

53 In this report, we seek to better understand how uropod location of PIPKI $\gamma 90$ is controlled in T

54 cells, and its role in T cell uropod formation. By over-expressing GFP-tagged PIPKI $\gamma$ in murine

55 D10 T cells and in human freshly isolated T cells, we find that the 26 amino acid extension of

56 PIPKI $\gamma 90$ is required for its localization to the uropod. We moreover observe that PIPKI $\gamma 90$

57 deficient murine T cells and human T cells expressing kinase-dead PIPKI $\gamma 90$ have elongated

58 uropods. These findings show that similar mechanisms control uropod targeting of PIPKI $\gamma 90$ in

59 both neutrophils (Xu et al., 2010) and T cells (this work), and that PIPKI $\gamma 90$ is involved in

60 uropod retraction in both cell types. We also provide novel data on the interrelationship between

61 flotillins and PIPKI $\gamma 90$.

62 
4

63 Materials and Methods

64 Ethics Statement

65 We received specific approval for this study from the University of Wisconsin School of

66 Medicine and Public Health Institutional Animal Care and Use Committee (protocol mumber

67 MO1570-0-06-07).

68

69 Mice

70 PIPKI $\gamma 90^{-/-}$mice were generated as previously described and fully backcrossed onto the

71 C57/Black6 background (Wernimont et al., 2010).

72

73 Reagents

74 Antibodies for immunofluorescence and immunoblotting:

75 Phospho ezrin (Thr567)/ radixin (Thr564)/ moesin (Thr558) (P-ERM) (Cell Signaling

76 Technology, Beverly, MA); flotillin-2 (Sigma, F-1680).

77 Recombinant proteins: human stromal cell-derived factor-1 (SDF-1 alpha), recombinant murine

78 intercellular adhesion molecule-1 (rmICAM-1) and recombinant human ICAM-1 (rhICAM-1)

79 were purchased from R and D systems (Minneapolis, MN).

80 Gey's solution contained $138 \mathrm{mM} \mathrm{NaCl}, 6 \mathrm{mM} \mathrm{KCl}, 100 \mu \mathrm{M}$ EGTA, 1 mM Na $\mathrm{HPO}_{4}, 5$ mM

$81 \mathrm{NaHCO}_{3}, 5.5 \mathrm{mM}$ glucose and $20 \mathrm{mM}$ HEPES (pH 7.4).

82

83 Isolation, cell culture and retroviral transfection of murine T cells

84 Murine D10 T cells (ATCC) were retrovirally transduced with GFP or GFP-PIPKI $\gamma 87$,

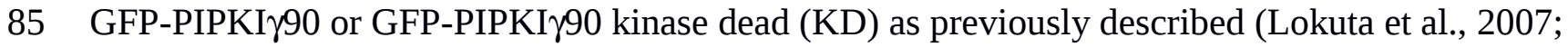

86 Wernimont et al., 2010). Following retroviral transduction, fluorescent cells were sorted by

87 FACS. T cells were maintained in complete RPMI supplemented with IL-2 (Chiron).

88 Single cell suspensions of primary mouse T cells were made from lymph nodes and spleen from

89 control and PIPKI $\gamma 90$ knockout mice that were between 6 and 10 weeks of age. Following red

90 blood cell lysis, CD4+ T cells were isolated from cell suspension by negative selection and 
5

91 automacs sorting (Miltenyi). Isolated CD4+ T cells were then stimulated 1:1 with

92 anti-CD3/CD28 coated beads according to manufacturer's instructions (Invitrogen) and

93 maintained in RPMI supplemented with IL-2 (Chiron). These anti-CD3/CD28 bead activated

94 cells were used for in vitro assays days 7 to 10 following isolation.

95

96 Isolation and transient transfection of human T cells

97 T-lymphocytes were isolated from buffy coats of healthy donor blood using the Pan T Cell

98 Isolation Kit II (Miltenyi Biotec) and separation on LD columns (Miltenyi Biotec) according to

99 the manufacturer's instructions. The buffy coats were obtained from the Central Laboratory of the

100 Swiss Red Cross, Bern, Switzerland. For details see Affentranger et al. (2011).

101 For transient transfections, $5 \times 10^{6}$ freshly isolated $\mathrm{T}$ cells were resuspended in $100 \mu \mathrm{l}$ human $\mathrm{T}$

102 cell nucleofector solution (Amaxa, Köln, Germany) diluted 1:2 with PBS and 1-2 $\mu$ g of DNA per

103 construct was added, followed by nucleofection (Amaxa Nucleofector, program U-14).

104 Constructs encoding for flotillin-2 and -1 C-terminally tagged with mCherry were prepared as

105 described (Rossy et al., 2009; Affentranger et al., 2011). Constructs encoding for PIPKI $\gamma 90$ and

106 PIP5K 887 N-terminally tagged with GFP and subcloned in a pcDNA3.1 vector were prepared as

107 described (Lokuta et al., 2007). The point mutation D253A was introduced into the wild type

108 enzyme using the Quick Change Mutagenesis kit from Stratagene.

109 Immediately after transfection, $500 \mu \mathrm{l}$ of RPMI with $20 \%$ FCS was added and the cells were

110 transferred to a prewarmed 12-well plate containing $2.5 \mathrm{ml}$ of RPMI with 20\% FCS, followed by

111 incubation at $37^{\circ} \mathrm{C}$ in a $\mathrm{CO}_{2}$ incubator for 4-6 hours. Transfected cells were subsequently washed

112 and resuspended in Gey’s solution.

113

114 Immunofluorescence staining of murine T cells

115 Murine wild type and PIPKI $990^{-/-}$T cells were allowed to migrate on rmICAM-1 coated

116 coverslips for 15 minutes at $37^{\circ} \mathrm{C}$ prior to fixing with $3 \%$ paraformaldehyde (PFA) (Electron

117 Microscopy Services) for 15 minutes at $25^{\circ} \mathrm{C}$. Cells were permeabilized with $0.2 \%$ Triton X-100,

118 blocked in goat serum and stained with anti-P-ERM antibody (Santa Cruz), DAPI and rhodamine 
6

119 phalloidin (Invitrogen) along with FITC conjugated anti-rabbit secondary antibodies (Jackson

120 Labs). Images were acquired on a laser scanning confocal microscope (Olympus) using a 60X

121 Plan Apo/1.45 oil immersion objective with a 10x zoom factor and captured into Fluoview

122 software (FV10-ASW version 01.07; Olympus).

123 Immunofluorescence staining of human T cells

124 The transfected cells were resuspended in Gey’s medium containing $1 \mathrm{mM} \mathrm{MgSO} 4,1.1 \mathrm{mM}$

$125 \mathrm{CaCl}_{2}$ and plated on glass coverslips coated with $3 \mu \mathrm{g}$ hrICAM- $1 / \mathrm{ml}$, incubated at $37^{\circ} \mathrm{C}$ and $5 \%$

$126 \mathrm{CO}_{2}$ for 45 minutes followed by addition of SDF-1 (40 ng/ml) and a further incubation for 15

127 minutes. Cells were fixed with 3.7\% PFA for 15 minutes followed by staining with rhodamine

128 phalloidin (Molecular Probes) and Hoechst 33342 dye (Sigma) or with an antibody recognizing

129 flotillin-2 as described (Affentranger et al., 2011). Pictures were acquired on a confocal

130 microscope (Olympus FV 1000) equipped with an 60X Plan Apo/1.45 oil immersion objective

131 and captured into Fluoview software (FV10-ASW version 01.7).

132 Flotillin- and PIPKI 990 -enriched aggregates located at the plasma membrane of at least $1 \mu \mathrm{m}$ or 133 larger (maximally approximately $4 \mu \mathrm{m}$ ) were defined as “caps”. For analysis of shape and protein 134 localization, 50 - 100 T cells were analysed per sample and experiment.

135

136 Statistical analysis

137 Data were analyzed with the Graph Pad Prism software (version 5.04) using ANOVA with

138 Tukey's post hoc testing or with the student's t-test, depending on the experiment, as detailed in 139 the Figure legends. $P$ values $<0.05$ were considered significant. Data correspond to the mean \pm 140 sem. 
141 Results

142 PIPKI 900 specifically localizes to the T cell uropod independent of its kinase activity but

143 dependent on its 26 residue C-terminal extension

144 Previous work from our lab with murine T cells has identified PIPKI $\gamma 90$ as a uropod component

145 in neutrophils and murine T cells (Lokuta et al., 2007). We studied kinetics of uropod recruitment

146 of PIPKI 90 in human freshly isolated T cells stimulated with the chemokine SDF-1. As known T

147 cell uropod components we used the the raft proteins flotillin-1 and -2 (Affentranger et al., 2011;

148 Baumann, Affentranger \& Niggli, 2012). As shown in Figure 1A, flotillins and PIPKIү90 are

149 mostly uniformly distributed in the spherical resting cells, but cap rapidly and arrive together in

150 the uropod upon chemokine addition. Capping especially of flotillins precedes formation of the

151 uropod. Capping of PIPKI $\gamma 90$ lags slightly behind that of flotillins (t1/2 for flotillin:

152 approximately 1 minute; t1/2 for PIPKIү90: approximately 2 minutes), and the percentage of

153 fully polarized cells with flotillin caps is higher than that of cells with PIPKIү90 caps. (Figure

154 1B).

155 We now studied mechanisms involved in uropod targeting of this enzyme by expressing

156 GFP-tagged wild type and mutated PIPKI $\gamma 90$ in freshly isolated human T cells. The constructs

157 used in this study are shown in Fig. 2A. We found that wild type PIPKI 90 locates to the uropod

158 of freshly isolated human T cells randomly migrating on ICAM-1 in the presence of SDF-1 (Fig.

159 2B), as well as in murine D10 T cells migrating on ICAM-1 (supplementary Fig. S1).In

160 neutrophils the PIPKIY $90 \mathrm{kDa}$ isoform specifically localizes to the uropod while the $87 \mathrm{kDa}$

161 isoform is uniformly distributed around the cell cortex (Lokuta et al., 2007; Xu et al., 2010),

162 suggesting that its localization is regulated by the 26 residue C-terminal extension.

163 We obtained comparable results also in human T cells (Figure 2B,C). Kinase-dead PIPKI $\gamma 90$ also

164 localizes to the T cell uropod indicating that localization is not kinase dependent. (Figure 2B;C).

165 Similar data were obtained for murine D10 T cells (supplementary Fig. S1). In human T cells

166 expressing GFP-tagged PIPKI $\gamma 87$ and plated on ICAM-1 in the presence of the chemokine

167 SDF-1, formation of contracted uropods was almost completely abolished. Capping of the uropod

168 component flotillin-2 was also markedly reduced (Figure 2B,C). Similarly uropod capping of the 
8

169 adhesion receptor PSGL-1 was inhibited (V. Niggli and S. Affentranger, unpublished

170 observations). This inhibitory effect was less striking in the murine D10 T cell clone (Fig. S1),

171 possibly because these cells are already activated and polarized in the absence of stimuli, whereas

172 freshly isolated $\mathrm{T}$ cells are mainly spherical in the absence of chemokine (Figure 1A). In the latter

173 situation, transfection with the PIPKI 87 construct prevents polarization whereas for the D10 T

174 cell clone already existing uropods may be resistant to disruption. Our findings suggest a role of

175 PIPKI $\gamma 90$ in uropod formation, possibly as a scaffolding protein.

176

177 Transfection of human T cells with a dominant-negative mutant of flotillin-2 impairs capping of 178 PIPKI $\gamma 90$

179 We have previously shown, that flotillins are involved in T cell uropod formation and that 180 transfection of human T cells with a dominant-negative mutant of flotillin-2; flotillin-2-G2A

181 impairs uropod formation and capping of uropod proteins such as PSGL-1 (Affentranger et al., 182 2011). Similary, transfection of human T cells with this mutant significantly reduced

183 chemokine-induced capping of PIPKI $\gamma 90$ by $64 \pm 2 \%(n=3, p<0.001)$ (Figure 3A,B), suggesting 184 that flotillins and PIPKI $\gamma 90$ cooperate as scaffolding proteins in the uropod. Cell polarity was 185 also inhibited by $41 \pm 2 \%(n=3, p<0.01)$ in SDF-1-stimulated cells, in agreement with previous 186 data on human T-lymphoblasts (Affentranger et al., 2011).

187

188 189 PIPKI $90^{-/}$murine T cells or human T cells expressing kinase-dead PIPKI 90 have elongated uropods

190 Given the specific localization of PIPKI $\gamma 90$ to the T cell uropod, and the negative impact of

191 PIPKI $\gamma 87$ on uropod formation in human T cells, we explored the role of PIPKI $\gamma 90$ in uropod 192 formation using CD4+ T cells from PIPKI $\gamma 90^{-/-}$mice. These cells were fixed while migrating on 193 ICAM-1 and stained with antibodies recognizing phosphorylated ERM proteins as uropod 194 markers along with DAPI and rhodamine phalloidin to visualize the nucleus and actin 195 cytoskeleton respectively. As shown in Fig. 4, T cells from PIPKI $\gamma 90$ knockout mice were longer 
9

196 than control cells. Quantification of the cell body length, defined as the length from the leading

197 edge to the trailing edge of the nucleus, was not different between control and knockout cells.

198 Rather, the difference in cell length was attributable to an approximately 30\% increase in uropod

199 length (measured from the trailing edge of the nucleus to the end of the uropod).

200 As shown in Fig. 2, expression of kinase-dead PIPKIy90 in human T cells did not affect cell

201 polarization and capping of uropod proteins such as flotillins. However during SDF-1-stimulated

202 migration on ICAM-1, freshly isolated human T cells transfected with kinase-dead PIPKI $\gamma 90$ had

203 elongated uropods as compared to cells expressing wild type PIPKI $\gamma 90$ or EGFP. We observed a

204 27\% increase in uropod length when comparing human T cells transfected with EGFP to cells

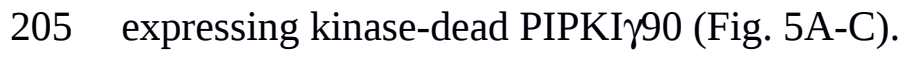

206

207 
10

\section{Discussion}

209 The work presented here shows that PIPKI $\gamma 90$ is specifically localized to the T cell uropod,

210 whereas the PIPKI $\gamma 87$ isoform shows a diffuse location, implicating the 26 amino acid

211 C-terminal extension in uropod targeting, similar to findings in neutrophils. Additionally, we 212 show that PIPKI $\gamma 90$ regulates adhesion of the T cell uropod since in its absence murine T cells

213 have elongated uropods, very likely due to impaired deadhesion of the rear of the cell.

214 Comparable findings were obtained for human T cells transfected with kinase dead PIPKI $\gamma 90$.

215 Moreover our data suggest novel scaffolding functions of PIPKI $\gamma 90$ in the uropod.

216 Our data indicate divergent functions for the 87 and 90 kDA PIPKI $\gamma$ isoforms. This is not entirely

217 unexpected, since previous work has shown that they differently regulate calcium signaling and

218 integrin mediated adhesion. For instance, PIPKI $\gamma 87$ is the isoform responsible for generating the

$219 \mathrm{PI}(4,5) \mathrm{P}_{2}$ required for calcium signaling (Wang et al., 2004), whereas specific knockdown of

220 PIPKI $\gamma 90$ actually increases adhesion of T cells to ICAM-1 (Wernimont et al., 2010).

221 We investigated the functional role of PIPKI $\gamma 90$ in uropod formation and show that murine T

222 cells lacking this enzyme or human T cells expressing kinase-dead PIPKI $\gamma 90$ have elongated

223 uropods when migrating on ICAM-1, comparable to differentiated neutrophil-like HL-60 cells

224 transfected with kinase-dead PIPKI $\gamma 90$ (Lokuta et al., 2007). This suggests that deadhesion of

225 uropods is impaired in cells lacking PIPKI $\gamma 90$ or expressing the kinase-dead mutant which may

226 interfere with the function of the endogenous enzyme, for example by displacing it from

227 interaction partners. Localized increases in $\mathrm{PI}(4,5) \mathrm{P}_{2}$ in the rear of the cell mediated by PIPKI $\gamma$

228 could lead to localized activation of ERM proteins, resulting in F-actin-membrane linkage and

229 enhanced Rho activity inducing uropod contraction (Ivetic \& Ridley, 2004). Knockdown of

230 PIPKI $\gamma$ could thus result in a reduction of uropod contractility and therefore a decrease in uropod

231 detachment.

232 Interestingly, expression of PIPKIy87, which lacks the uropod targeting domain, in human T cells

233 resulted in almost complete impairment of uropod formation and capping of uropod proteins (Fig.

234 2). These findings are similar to those previously obtained for the isoform PIPKI $\beta$ in 
11

235 neutrophil-like HL-60 cells (Lacalle et al., 2007). There, expression of kinase-dead PIPKI $\beta$

236 induced cell elongation, whereas expression of a truncated form of PIPKI $\beta$ lacking the last 83

237 C-terminal amino acids, which does not locate to the uropod, had a much stronger phenotype and 238 reduced cell polarization by about 80\%. Lacalle et al. (2007) argue that the truncated PIPKI $\beta$ may

239 act by sequestering proteins required for uropod formation and prevent their interaction with 240 endogenous PIPKI $\beta$, thus strongly impairing uropod formation. This could also explain the strong 241 effects of the mutant PIPKI 87 observed in this work. In the murine T cells lacking PIPKI $\gamma 90$, 242 other isoforms such as PIPKI $\beta$ could compensate for this loss and explain the relatively mild 243 phenotype. Transfection with the kinase-dead PIPKI $\gamma 90$ may only incompletely interfere with 244 activity of the endogenous PIPKI $\gamma$ isoform. Both PIPKI $\gamma 90$ and PIPKI $\beta$ may thus act as scaffolds 245 to organize signaling at the rear of polarized leukocytes, together with the raft-associated 246 flotillins. We show that flotillins, directly or indirectly, also are involved in PIPKI 90 uropod 247 targeting (Figure 3). PIPKI $\gamma 90$ and PIPKI $\beta$ have divergent C-terminal amino acid sequences and 248 may be targeted to the uropod via different mechanisms. For example, PIPKI $\gamma 90$, but not PIPKI $\beta$, 249 interacts with talin as shown in vitro (Manes et al., 2010).

250

\section{Conclusions}

252 This work identifies a region of PIPKI 90 necessary for its uropod localization in T cells, shows 253 that T cells lacking PIPKI $\gamma 90$ or expressing kinase-dead PIPKI $\gamma 90$ have increased uropod length 254 similar to findings in neutrophils, implicates flotillins in uropod targeting of PIPKI $\gamma 90$ and 255 provides novel data on possible scaffolding functions of PIPKI $\gamma$.

256

\section{Acknowledgements}

258 We thank Kyle Legate for the PIPKI $\gamma 90^{-/-}$mice and Dr. E. Sigel for critical reading of the 259 manuscript. This work was supported by the Swiss National Science Foundation grant No 260 31003A_129655 to V.N. 
12

262 References

263 Baumann T, Affentranger S, Niggli V. 2012. Evidence for chemokine-mediated coalescence of 264 preformed flotillin hetero-oligomers in human T-cells. Journal of Biological Chemistry 265 287:39664-39672.

266

267 Affentranger S, Martinelli S, Hahn J, Rossy J, Niggli V. 2011. Dynamic reorganization of 268 flotillins in chemokine-stimulated human T-lymphocytes. BMC Cell Biology 12:28.

269

270 Bairstow SF, Ling K, Su X, Firestone AJ, Carbonara C, Anderson RASF. 2006. Type Igamma661 271 phosphatidylinositol phosphate kinase directly interacts with AP2 and regulates endocytosis.

272 Journal of Biological Chemistry 281:20632-20642.

273

274 Bolomini-Vittori M, Montresor A, Giagulli C, Staunton D, Rossi B, Martinello M, Constantin G, 275 Laudanna C. 2009. Regulation of conformer-specific activation of the integrin LFA-1 by a 276 chemokine-triggered Rho signaling module. Nature Immunology 10:185-194. 277

278 Calderwood DA, Tai V, Di Paolo G, De Camilli P, Ginsberg MH. 2004. Competition for talin 279 results in trans-dominant inhibition of integrin activation. Journal of Biological Chemistry 279: 280 28889-28895.

281

282 Ivetic A, Ridley AJ. 2004. Ezrin/radixin/moesin proteins and Rho-GTPase signaling in 283 leucocytes. Immunology 112:165-176.

284

285

286

287

Lacalle RA, Peregril RM, Albar JP, Merino E, Martinez-A C, Merida I, Manes S. 2007. Type I

phosphatidylinositol 4-phosphate 5-kinase controls neutrophil polarity and directional movement. Journal of Cell Biology 179:1539-1553.

288 
13

289 Lokuta MA, Senetar MA, Bennin DA, Nuzzi PA, Chan KT, Ott VL, Huttenlocher A. 2007. Type

290 Igamma PIP kinase is a novel uropod component that regulates rear retraction during neutrophil

291 chemotaxis. Molecular Biology of the Cell 18:5069-5080.

292 Ludwig A, Otto GP, Riento K, Hams E, Fallon PG, Nichols BJ. 2010. Flotillin microdomains

293 interact with the cortical cytoskeleton to control uropod formation and neutrophil recruitment.

294 Journal of Cell Biology 191:771-781.

295

296 Manes S, Fuentes G, Peregril RM, Rojas AM, Lacalle RA. 2010. An isoform-specific

297 PDZ-binding motif targets type I PIP5 kinase beta to the uropod and controls polarization of 298 neutrophil-like HL60 cells. FASEB Journal 24:3381-3392.

299

300 Rossy J, Schlicht D, Engelhardt B, Niggli V. 2009. Flotillins interact with PSGL-1 in neutrophils 301 and, upon stimulation, rapidly organize into membrane domains subsequently accumulating in 302 the uropod. PLoS One 4:e5403.

303

304 Sánchez-Madrid F, Serrador JM. 2009. Bringing up the rear: defining the roles of the uropod.

305 Nature Reviews of Molecular Cell Biology 10:353-359.

306

307 Sekine Y, Tsuji S, Ikeda O, Sugiyma K, Oritani K, Shimoda K, Muromoto R, Ohbayashi N, 308 Yoshimura A, Matsuda T. 2007. Signal-transducing adaptor protein-2 regulates integrin-mediated 309 T cell adhesion through protein degradation of focal adhesion kinase. Journal of Immunology 310 179:2397-2407.

311

312 Serrador JM, Alonso-Lebrero JL, del Pozo MA, Furthmayr H, Schwartz-Albiez R, Calvo J,

313 Lozano F, Sanchez-Madrid F. 1997. Moesin interacts with the cytoplasmic region of intercellular 314 adhesion molecule-3 and is redistributed to the uropod of T lymphocytes during cell polarization. 315 Journal of Cell Biology 138:1409-1423.

316 
14

317 Soriano SF, Hons M, Schumann K, Kumar V, Dennier TJ, Lyck R, Sixt M, Stein JV. 2011. In vivo 318 analysis of uropod function during physiological T cell trafficking. Journal of Immunology

319 187:2356-2364.

320

Sun Y, Thapa N, Hedman AC, Anderson RA. 2013. Phosphatidylinositol 4,5-bisphosphate:

322 targeted production and signalling. Bioessays 35: 513-522.

323

324

325

326

327

Ward SG, Westwick J, Harris S. 2011. Sat-Nav for T cells: Role of PI3K isoforms and lipid

328

phosphatases in migration of T lymphocytes. Immunology Letters 138:15-18.

329

330

Wernimont SA, Legate KR, Simonson WTN, Fässler R, Huttenlocher A. 2010. PIPKI gamma 90

331

negatively regulates LFA-1-mediated adhesion and activation in antigen-induced CD4+ T cells.

332

Journal of Immunology 185:4714-4723.

333

334

Xu J, Wang F, Van Keymeulen A, Herzmark P, Straight A, Kelly K, Takuwa Y, Sugimoto N,

335

Mitchison T, Bourne HR. 2003. Divergent signals and cytoskeletal assemblies regulate

336

self-organizing polarity in neutrophils. Cell 114:201-214.

337

338

Xu W, Wang P, Petri B, Zhang Y, Tang W, Sun L, Kress H, Mann T, Shi Y, Kubes P, Wu D. 2010.

339

Integrin-induced PIP5K1C kinase polarization regulates neutrophil polarization, directionality,

340 and in vivo infiltration. Immunity 33: 340-350.

341

342

343 


\section{Figure 1}

Figure 1. PIPKI g 90 cocaps with flotillins in human T cells during SDF-1-induced uropod formation.

T Cells were co-transfected with GFP-tagged wild type PIPKI g 90, mCherry-flotillin-1-mCherry and flotillin-2-mCherry, followed by incubation at $37^{\circ} \mathrm{C}$ for 30 minutes in suspension. Note that singly expressed flotillin-1 or -2 do not cap (Affentranger et al., 2011) . Cells were then treated with SDF-1 (40 ng/ml) for the indicated times and fixed in suspension with 3,7\% PFA. (A) Fluorescence pictures. Scale bar $10 \mu \mathrm{m}$. (B) Quantitative evaluation of (A). The percentage of transfected cells with capped flotillins or PIPKIy90 were derived from $\mathrm{n}=3$ independent experiments (mean $\pm \operatorname{sem})(* \mathrm{p}<0.05$, ** $\mathrm{p}<0.01 * * * \mathrm{p}<0.001, * * * * \mathrm{p}<0.0001$ for differences to cells not exposed to SDF-1, obtained with Anova and Tukey's multiple comparison test). 50 cells were counted per sample and experiment. 


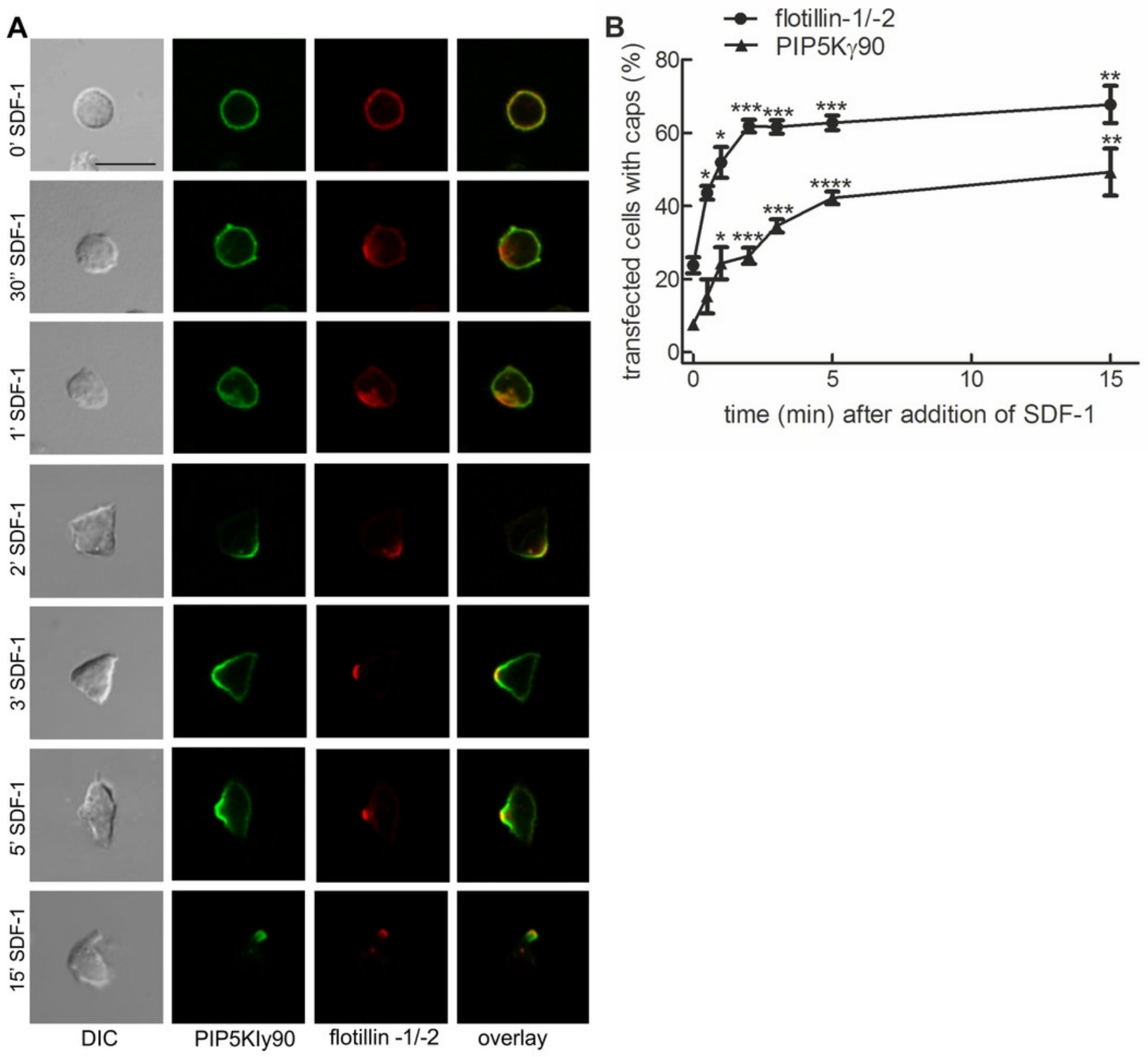

Figure 1 


\section{Figure 2}

Figure 2. Determinants of uropod targeting of PIPKI g 90 in human T cells.

(A) Scheme showing the structure of PIPKI g 90 and of the constructs used in this work. (B,C) Human freshly isolated T cells were transiently transfected with GFP-tagged wild type or the indicated mutant PIPKI g constructs, followed by plating on ICAM-1, and, after a 45 min incubation at $37^{\circ} \mathrm{C}$, stimulation with $40 \mathrm{ng}$ SDF-1/ml for 15 min. Cells were then fixed with PFA and stained for endogenous flotillin-2 (flo2) with a rabbit polyclonal antibody. (B) Representative immunofluorescence pictures showing the location of GFP tagged wild type and mutant PIPKI g (green) and flotillin-2 (red). Scale bar $10 \mu \mathrm{m}$. (C) Quantitative evaluation of the experiment shown in (B) concerning the \% of transfected polarized cells and the \% of transfected cells with PIPKI g and flotillin-2 (flo2) caps. Mean \pm sem of 3 experiments $\left({ }^{* *} \mathrm{p}<0.01\right.$ as compared to cells transfected with wild type PIPKI g 90 obtained by ANOVA and Tukey's multiple comparison test). 50 cells were counted per sample and experiment. 


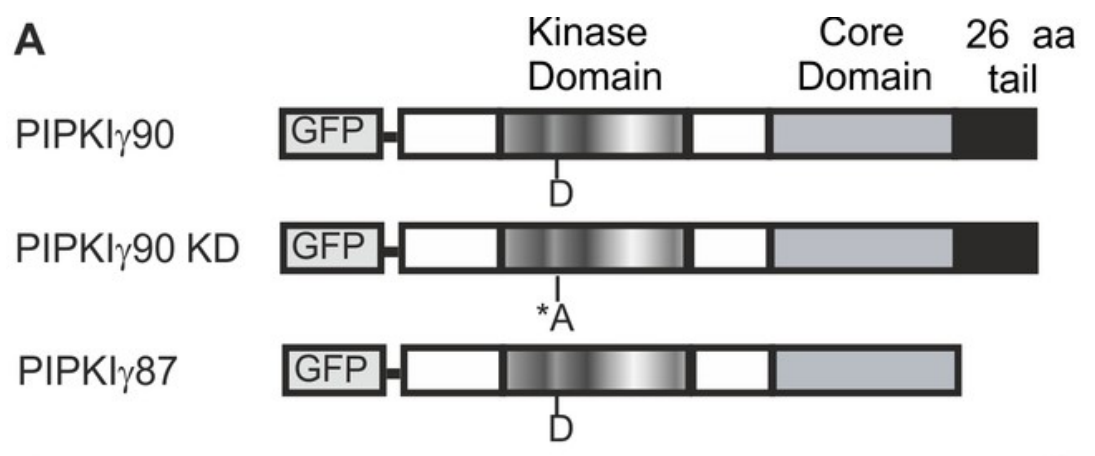

B

PIPKl $\gamma 90$

PIPKI 90 KD

PIPKl $\gamma 87$
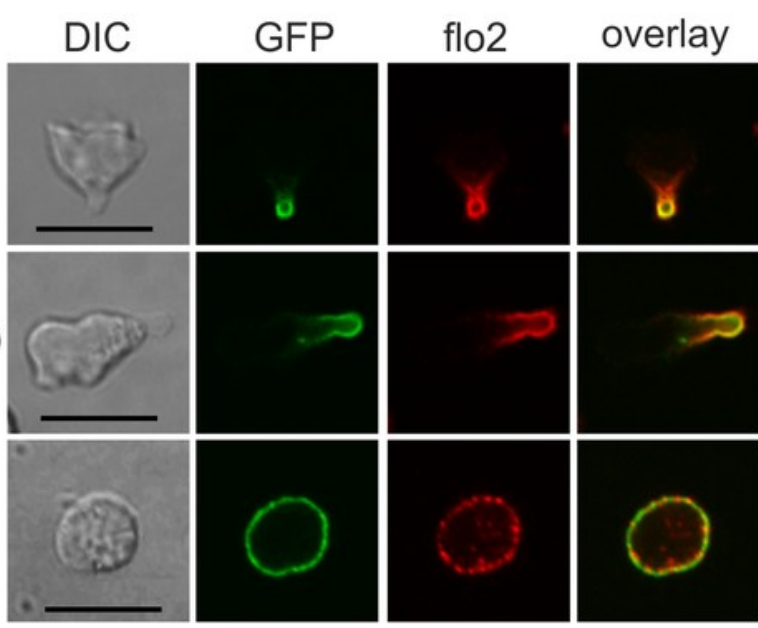

C $\square$ polarized cells

cells with PIPKl $\gamma$ caps cells with flo2 caps

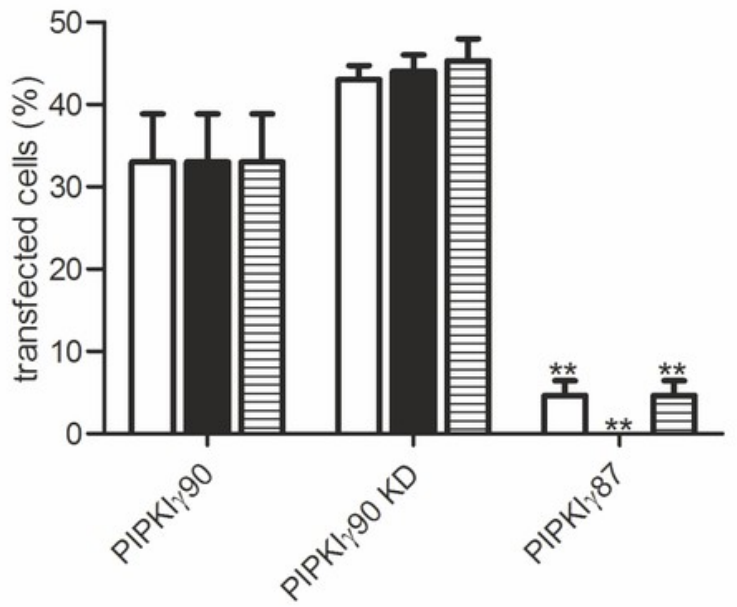

\section{Figure 2}




\section{Figure 3}

Figure 3. Coexpression of dominant-negative flotillin-2-G2A inhibits capping of GFP-PIPKIy90 in human T cells.

T cells were co-transfected with wild type flotillin-2-HA and GFP-tagged wild type PIPKI g 90 or flotillin-2-G2A-HA and GFP-tagged wild type PIPKI g 90 and incubated at $37^{\circ} \mathrm{C}$ for 30 minutes. Cells were then stimulated with SDF-1 (40 ng/ml) for 15 minutes, fixed with 3,7\% PFA (final concentration) in suspension and stained for HA. (A) Fluorescence pictures. Scale bar $10 \mu \mathrm{m}$. (B) Quantitative evaluation of A: \% of cells with PIPKI g 90 caps in transfected cells were derived from n=3 independent experiments (mean \pm sem) $\left({ }^{* * *} \mathrm{p}<0.001\right.$ obtained by ANOVA and Tukey's multiple comparison test). 100 cells counted per sample. 
A

no SDF-1

DIC PIPKly90 flo2-HA overlay

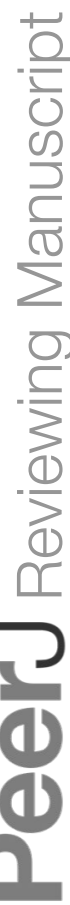

B

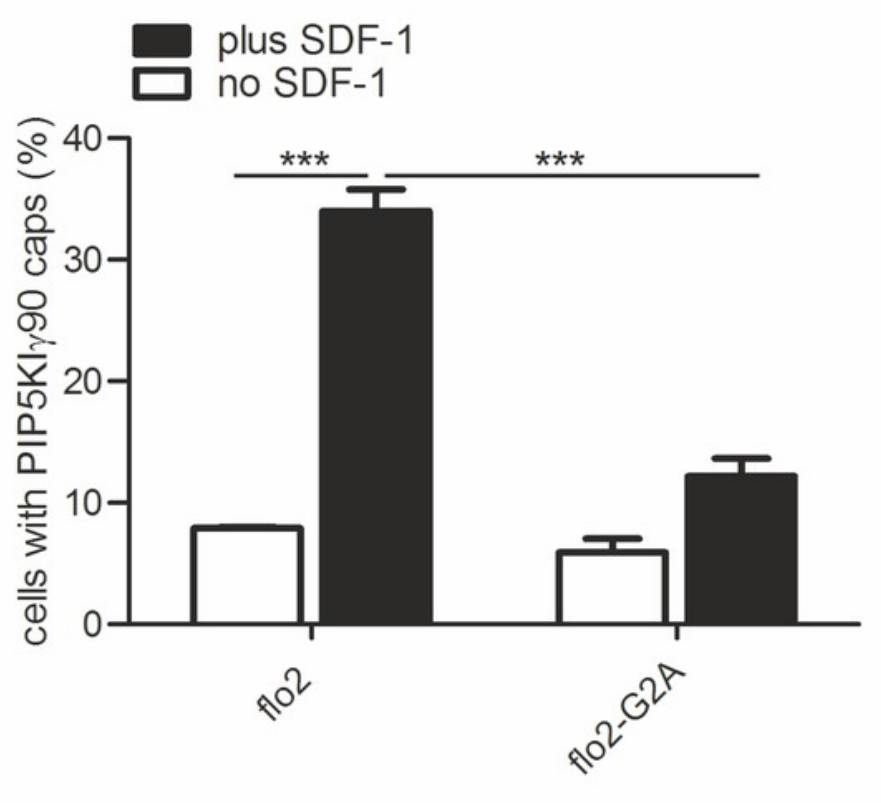

\section{Figure 3}




\section{Figure 4}

Figure 4. Murine PIPKI g $90^{-/-}$T-cells have elongated uropods.

Wild type and knockout murine T cells were fixed while migrating on an ICAM-1 coated coverslip and stained with DAPI, rhodamine phalloidin, and antibodies specific for P-ERM. (A) Representative immunofluorescence pictures showing the location of DAPI (blue), F-actin (red) and P-ERM (green).

Scale bar $10 \mu \mathrm{m}$. (B) Quantification of cell body length defined as the distance in $\mu \mathrm{m}$ from the leading edge to the trailing edge of the nucleus. (C) Quantification of uropod length-defined as the length in $\mu \mathrm{m}$ from the trailing edge of the nucleus to end of the uropod which is enriched in P-ERM. ${ }^{*}=\mathrm{p}<0.001$ by students $t$ test (mean \pm sem from 40 cells per condition from 3 independent experiments).

A

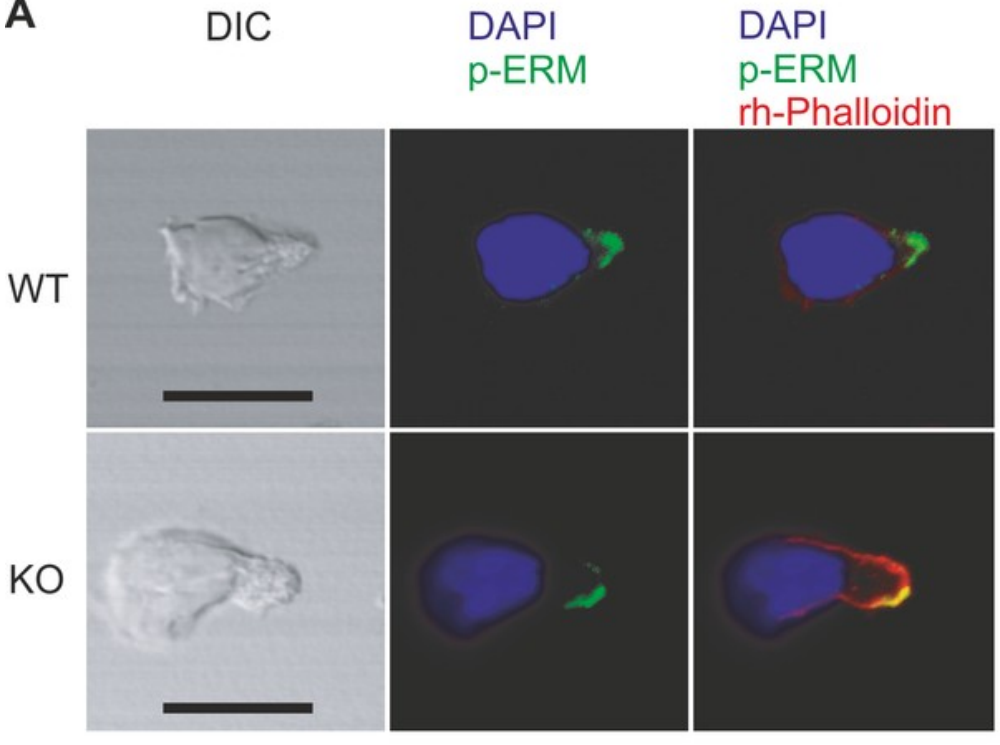

B

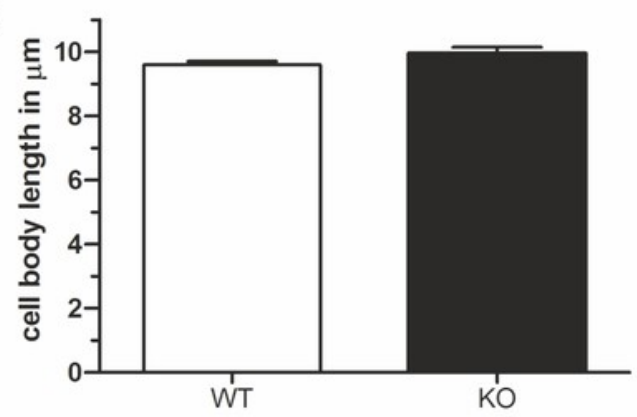

C

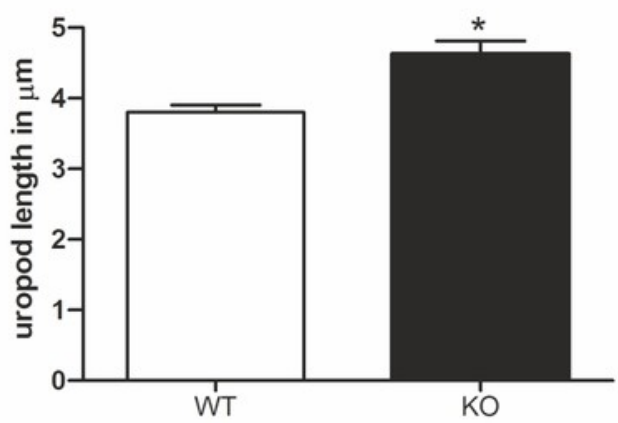




\section{Figure 5}

Figure 5. Transfection with kinase-dead PIPKI g 90 induces uropod elongation in human T cells.

Human T cells were transiently transfected with GFP, GFP tagged wild type PIPKI g 90, or GFP tagged kinase dead PIPKI g 90 (PIPKI g $90 \mathrm{KD}$ ). Cells were incubated at $37^{\circ} \mathrm{C}$ for 30 minutes, incubated on ICAM-1-coated cover slips at $37^{\circ} \mathrm{C}$ for 45 minutes, stimulated with SDF-1 (40 ng/ml) for 15 minutes, fixed with 3.7\% PFA and stained with Hoechst dye and rhodamine phalloidin. (A) Representative immunofluorescence pictures showing the location of the PIPKI g constructs (green), Hoechst dye (blue) and F-actin (red). Scale bar $10 \mu \mathrm{m}$. ( B) Quantification of cell body length-defined as the distance in $\mu \mathrm{m}$ from the leading edge to the trailing edge of the nucleus. (C) Quantification of uropod length-defined as the length in $\mu \mathrm{m}$ from the trailing edge of the nucleus to end of the uropod. Data shown in (B) and (C) were derived from a total of 60 cells per condition obtained in 3 independent experiments (mean $+/$ - sem) $\left({ }^{* *} \mathrm{p}<0.01\right.$ obtained by ANOVA and Tukey’s multiple comparison test). 
A

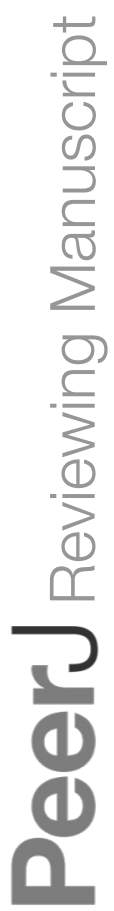

DIC

HOECHST HOECHST

GFP

GFP

rh-Phalloidin

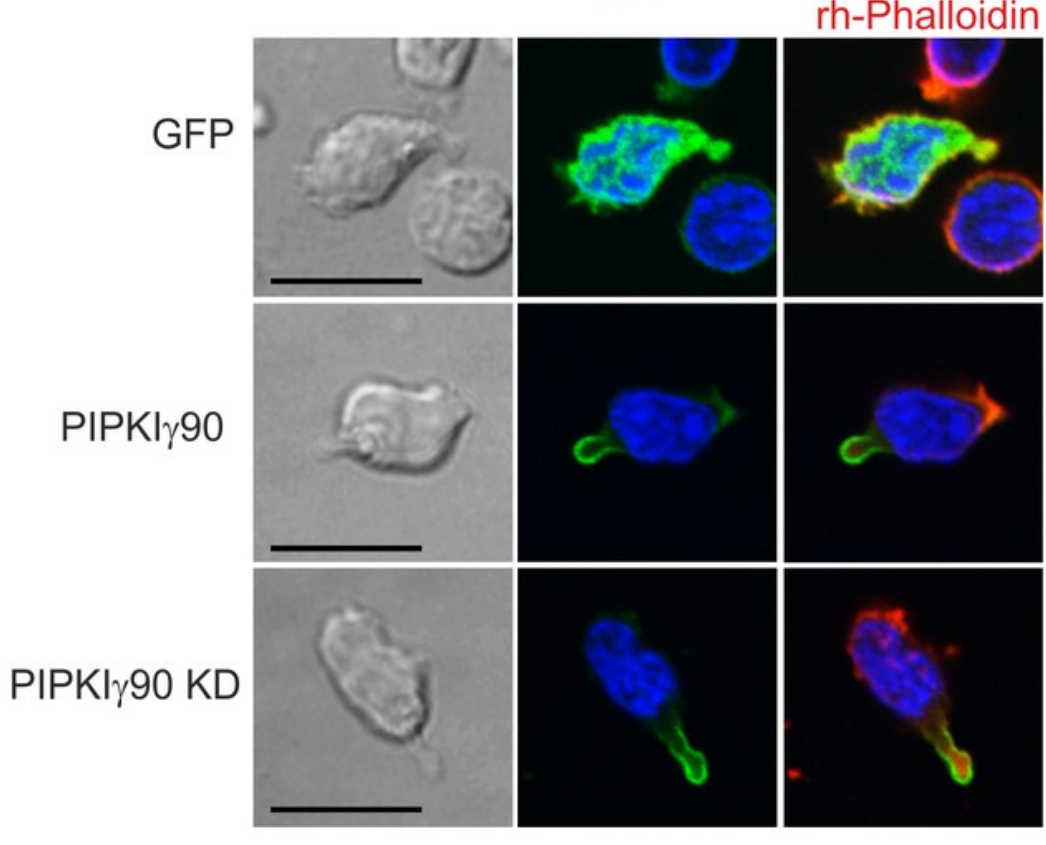

B

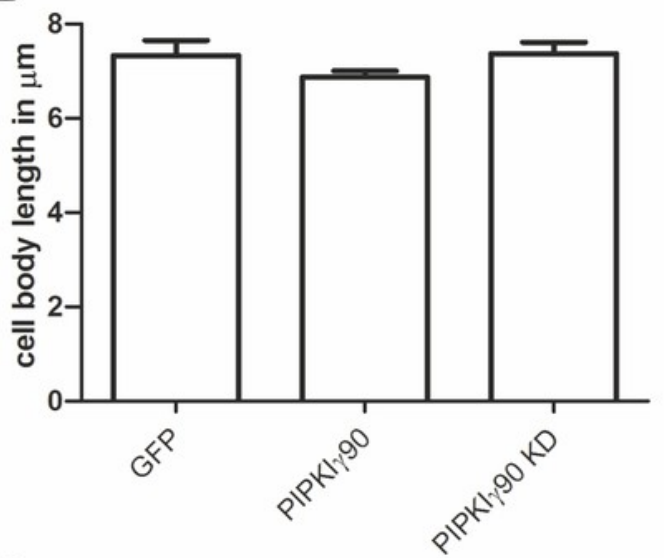

C

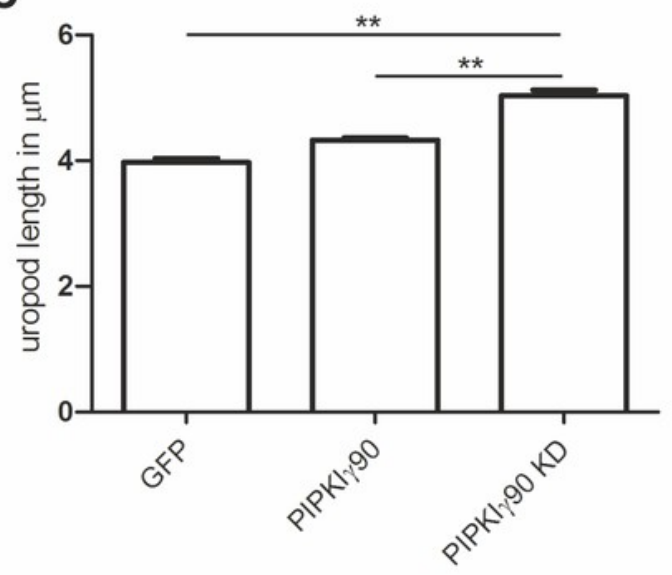

Figure 5 